\title{
Evolution of the martensitic transformation in shape memory alloys under high strain rates
}

\author{
D. Saletti ${ }^{1, a}$, S. Pattofatto ${ }^{1}$, and H. Zhao ${ }^{1}$ \\ Laboratoire de Mécanique et Technologie (LMT-Cachan), ENS-Cachan/CNRS-UMR8535/Université \\ Paris 6, 61 avenue du Président Wilson, F-94235 Cachan Cedex, France
}

\begin{abstract}
The specific properties of the shape memory alloys are mainly due to the martensitic transformation occuring in the material when mechanical or thermal loadings are applied. Here, the effect of strain rate on the transformation on an NiTi SMA is studied in tension. Different tests were performed at different strain rates in the range of $0,0001 / \mathrm{s}$ to $15 / \mathrm{s}$. Two distinct methods were used to measure the extension rate of the martensitic phase region in the specimen: digital image correlation technique and infrared thermography (IR during quasi-static tensile tests only). For the dynamic tensile tests, a Split Hopkinson Tensile Bar set-up was used with a fast camera recording at 45'000 fps. A superimposition of DIC and IR measurements in time and space can be done during quasi-static tests and results show that the temperature peak, as expected, follows the transformation front. As a consequence of the former validation of the DIC procedure, the velocity of the transformation front at high strain rate is deduced from space-time figures. As a conclusion, in the range of strain rates investigated in this paper, no strain rate sensitivity is observed for dynamics of extension of the transformation region.
\end{abstract}

\section{Introduction}

Due to two of their specific properties, namely superelasticity and the shape memory effect, shape memory alloys (SMA) have a great potential of applications in a lot of innovative technologies. In particular, the NiTi-based SMA are widely used as biomaterials (to manufacture stents for heart artery for instance) or in mechanical applications such as in the automotive industry, household appliances, and many other everyday goods.

In such SMA, two phases can be found, separately or combined. The transformation from a phase to the other is triggered when the material is undergoing variation of temperature or mechanical sollicitation, or both. By analogy with steel, the transformation, the mother phase and the daughter phase are respectively called martensitic transformation, austenite phase and martensite phase. In the case of SMA, martensitic transformation is reversible. Superelastic properties of the SMA are shown on a stress-strain curve, which is decomposed in three parts: first the elastic response of the material in a full austenite phase, then generally a plateau where martensitic transformation is occuring and propagating, which is visible through imaging techniques ([1]), and finally the homogeneous elastic response of the material in a full-martensitic phase. Studies have already been done on the influence of strain rate on the macroscopic behaviour of the SMA ([2]). The higher the strain rate, the more the slope of the plateau part of the stress-strain curve raises. This can be found in compression as well as in tension, despite the asymmetry between the two modes of loading. Assumptions have been made to explain the change in high strain rate response ([3]) and refer to the adiabatic regime at the transformation front, preventing the austenite phase from tranforming unless the level of stress is raised.

a e-mail: saletti@lmt.ens-cachan.fr

This is an Open Access article distributed under the terms of the Creative Commons Attribution-Noncommercial License 3.0, which permits unrestricted use, distribution, and reproduction in any noncommercial medium, provided the original work is properly cited. 
In this paper the effect of strain rate on the martensitic transformation in tension on a NiTi SMA is studied. Different tests were performed at different strain rates in the range of $10^{-4} \mathrm{~s}^{-1}$ to $15 \mathrm{~s}^{-1}$, extending the range of observations of the investigations previously done by [4]. Methods were used to measure the velocity of the propagation of the martensitic phase in the specimen: digital image correlation (DIC) techniques and infrared thermography (IR, during the quasi-static tensile tests only).

\section{Experimental techniques}

The measurement techniques employed in this study were different depending on the strain rate applied. For the $1.67 \cdot 10^{-4} \mathrm{~s}^{-1}$ strain rate, a classical electromechanical INSTRON machine was used associated with a reflex camera recording at 0.25 frame per second (fps) and an IR thermography device. For the $1.67 \cdot 10^{-2} \mathrm{~s}^{-1}$ strain rate, the same machine was used but with a fast camera recording at $125 \mathrm{fps}$. For the $16 \mathrm{~s}^{-1}$ strain rate which corresponds to the dynamic tensile test, a Split Hopkinson Tensile Bar (SHTB) set-up was used with a fast camera recording at 45'000 fps.

\subsection{Specimen characteristics}

A single NiTi plate-shape specimen was used for the whole of the experiments. The dimensions are $60 \times 3 \times 1.2 \mathrm{~mm}^{3}$. Tensile tests were performed at three different strain rate ranges and at room temperature, clearly above the characteristic $A_{f}$ temperature so that for stress-free condition the specimen is in a full austenite state. For all the experiments, the specimen is fixed between two tabs that can further be screwed (i) in the bars for dynamic tensile experiment, (ii) in a clamping system for static experiments. This allows to be more confident in the comparison of experimental results obtained with different devices.

In order to plot a behaviour curve of the material, a simple tension experiment was performed on the same specimen, after the experimental series of dynamic and static tests presented on the following article being completed. Experiment was conducted on an electro-mechanical testing machine at a prescribed velocity of $0.01 \mathrm{~mm} / \mathrm{s}$. Nominal stress-strain curve is shown in figure 1 . Three domains appear on the curve :

- elastic tension in the initial austenite for strains up to 0.01 ,

- a stress plateau associated to the superelastic domain of interest, corresponding to the stressinduced martensitic (SIM) transformation for strains up to 0.06. The stress at the beginning of the SIM transformation is called the transition stress. An unloading-reloading cycle has been done in the superelastic domain in order to show that the reverse transformation is effectively reversible,

- elastic tension in the supposed "fully" martensitic specimen. The notion of "fully martensitic" refers in fact to the end of the SIM transformation process even if it has been documented that in fact all austenite has not been transformed ([5]). In this experiment, maximal strain is under 0.08 and there is no evidence of plastic deformation during tension.

It can be noticed that two serrations are visible at the beginning of the superelastic domain. This phenomenon is due to the heterogeneity of the martensitic transformation and to the apparition of the martensitic zone.

\subsection{Measurement of dynamic nominal stress-strain curve}

Tension experiments at moderate impact velocitiy around $1 \mathrm{~m} / \mathrm{s}$ are performed on a tensile Hopkinson bar device. The moderate impact velocity allows to test the specimen in a dynamic regime for which pseudoelasticity still exists. At an initial impact velocity of $1 \mathrm{~m} / \mathrm{s}$, the strain rate is about $15 \mathrm{~s}^{-1}$. The Hopkinson bar allows to obtain an accurate measurement of forces and velocities on both sides of the specimen and subsequent deformation energy. So strains and displacements are given both by the Hopkinson bars and the optical digital image correlation (DIC) method applied to images acquired by the fast camera as explained in section 2.3.1. 


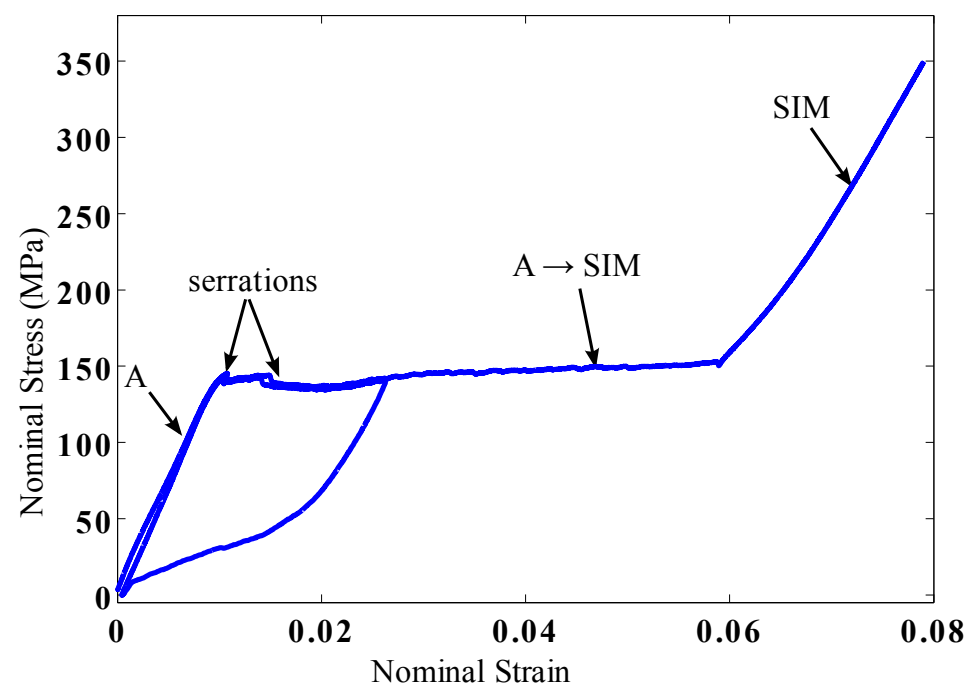

Fig. 1. Static strain-stress curve at nominal strain rate of $1.67 \cdot 10^{-4} \mathrm{~s}^{-1}$ at room temperature.

The device used in this study is a Split Hopkinson Tensile Bar (SHTB). It is composed of two bars called the input and the output bars, made of maraging steel. The specimen equipped with its end tabs is tightly screwed at the end of each bar. The tabs that are used to fix the specimen on the bars are manufactured in the same material of the bars and designed so as to minimize any mass effect by matching the same acoustic impedance as the bars. The experiment consists in creating a tensile longitudinal wave in the input bar, called the incident pulse, that propagates towards the specimen. When this pulse reaches the specimen, one part is reflected in the input bar while another part is transmitted by the sample to the output bar. Measurement of strain histories in several locations of the bars with strain gauges allows to determine force and particle velocitiy at both faces of the specimen ([6],[7]). This set-up is schematically presented in figure 2 , along with its dimensions. The incident pulse is obtained by sudden release of an initial elastic energy that is stored in the length $\mathrm{L}$ between points $\mathrm{A}$ and $\mathrm{B}$ of the input bar ([8]). The duration of the tension experiment being related to the length $\mathrm{L}$ and the base material of the bars, the test duration is in this case around $1500 \mu \mathrm{s}$. The initial stored energy depends on the initial load $N_{0}$ that is prescribed and controls the subsequent strain and strain rate applied to the specimen. To perform a test at an initial velocity of $1 \mathrm{~m} / \mathrm{s}$, a initial load of $1.5 \mathrm{kN}$ is applied.

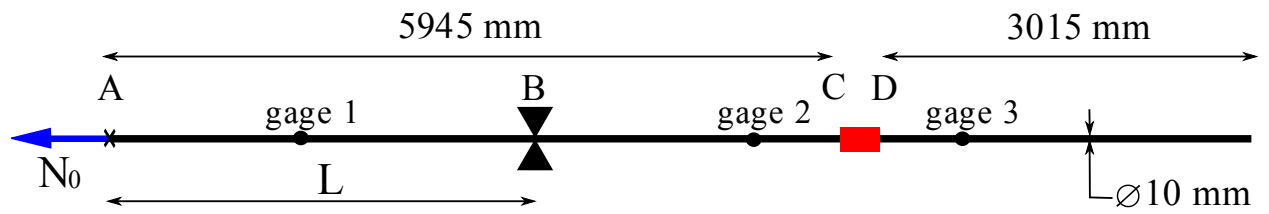

Fig. 2. Split Hopkinson Tensile Bar dimensions.

\subsection{Measurements of the heterogeneity of axial strain}

As mentionned in the introduction, pseudoelasticity in the NiTi alloy is due to a martensitic transformation that leads to a heterogeneous strain state in a tensile specimen, observation that is documented 
for static loadings. In order to follow this phenomenon at a tension velocity of $1 \mathrm{~m} / \mathrm{s}$, complementary measurements are conducted. Displacement and subsequent axial strain path along the specimen are calculated by digital image correlation (DIC). Moreover, since martensitic transformation is exothermic, infrared thermography (IRT) measurements are also a good mean to measure indirectly the location of the ongoing transformation. Optical and thermal measurements are obtained simultaneously on the same thin specimen, on two opposite sides that are supposed to have the same thermo-mechanical response. These two optical techniques are described in following sections, along with the experimental strategy that has been implemented.

\subsubsection{Digital Image Correlation (DIC) setup}

For dynamic experiments, a fast camera (Photron) is used to acquire images at a frame rate of $45^{\prime} 000$ fps with a resolution of $896 \times 64$ pixels, an integration time of $12 \mu$ s and a spatial resolution of $0.09 \mathrm{~mm} /$ pixel. For quasi-static tensile test, a reflex camera (Canon EOS) is used. The capture frequency is $0.25 \mathrm{fps}$ (one image every four seconds) in a full resolution of $2314 \times 3474$ pixels, that gives a spatial resolution of $0.026 \mathrm{~mm} /$ pixel. In order to allow DIC computations, a speckle was applied on the observed surface of the specimen with black and white paint. Moreover, to avoid heating of the specimen surface, the lighting was realized with LED lights during the quasi-static experiments and with short light exposition of the specimen during the dynamic experiments.

The principle of the DIC computation program (Correli ${ }^{\mathrm{Q}}$ ) that was used is given as follows ([9],elnasri07). Basically, pictures of the deforming specimen are taken during the experiments. The principle is to relate two images $f(\boldsymbol{x})$ and $g(\boldsymbol{x})$ corresponding to two different steps of loading, through a displacement field $\boldsymbol{u}$ to be calculated. Considering the conservation of the brightness, the following equation can be written, relating the image of reference $f(\boldsymbol{x})$ and the deformed image $g(\boldsymbol{x})$ through the unknown displacement $\boldsymbol{u}$ :

$$
g(\boldsymbol{x})=f[\boldsymbol{x}+\boldsymbol{u}(\boldsymbol{x})]
$$

Under the assumption that the image of reference is differentiable, the expression is developed in Taylor series, and the displacement field $\boldsymbol{u}$ comes as the result of the minimization of the following quadratic error:

$$
\eta^{2}=\iint[\boldsymbol{u}(\boldsymbol{x}) \cdot \nabla f(\boldsymbol{x})+f(\boldsymbol{x})-g(\boldsymbol{x})]^{2} d \boldsymbol{x}
$$

The last point of the specific Q4-method ([9]) is that the unknow displacement is decomposed over a set of scalar shape functions $\psi_{n}(\boldsymbol{x})$ as follows:

$$
\boldsymbol{u}(\boldsymbol{x})=\sum_{\alpha, n} a_{\alpha n} \psi_{n}(\boldsymbol{x}) \boldsymbol{e}_{\alpha}
$$

This leads to a linear system of equations that can be solved numerically through a spatial discretization in elements of any domain of interest selected in the image of reference. It has been documented that the performance of such a method is influenced by the size of the elements. Large size for the elements tends to improve the accuracy of the computed displacement field but to the detriment of spatial resolution. Then in the case of heterogeneous deformation, and in particular for localized deformation, the element size has to be a compromise between accuracy and spatial detection of the localized phenomenon. An element size around $30 \times 30$ pixels was found to be a good candidate in order to observe the transforming zone while limiting noise effects in the computations.

During dynamic tensile tests, the specimen exhibited large displacements. In this case, a strategy of update was used: the image of reference is regularly updated and the calculation of displacement field is performed by increments.

\subsubsection{Infrared Thermography (IRT) setup}

IRT pictures were acquired using an infrared camera (Jade CEDIP) with a resolution of $320 \times 240$ pixels. For every test, the frame period is $25 \mathrm{fps}$, the integration time to acquire a picture is $310 \mu \mathrm{s}$ and the spatial resolution is $0.27 \mathrm{~mm} /$ pixel. 
All the experiments were performed at a room temperature (around $300 \mathrm{~K}$ ). A thin layer of carbon black powder was deposited on the surface in order to make its emissivity higher and more homogeneous. Moreover, in order to improve the signal to noise ratio, and in particular to prevent effects of perturbative reflections, the optical path between the lens and the surface of the specimen was sheltered by a "tunnel cover" which inner surface was covered with black paint.

For the quasi-static tests, IRT measurements are given in addition to DIC measurements and used as a validation of the DIC technique for the measurement of transforming regions in the specimen. IRT thermography was not tried during dynamic tensile tests and this will be part of a further study.

\section{Analysis of the propagation velocity of the martensitic transformation on the quasi-static cases}

A first analysis of DIC results allows to give qualitative informations on the phenomena taking place during the tests. Figure 3, resulting from a quasi-static tensile test at $0.01 \mathrm{~mm} / \mathrm{s}$, shows two different strain zones: a $2 \%$ strain zone (supposed to be the austenitic phase) and a $6 \%$ strain zone (supposed to be the stress-induced martensitic phase). An enlargement of the SIM region can be observed.

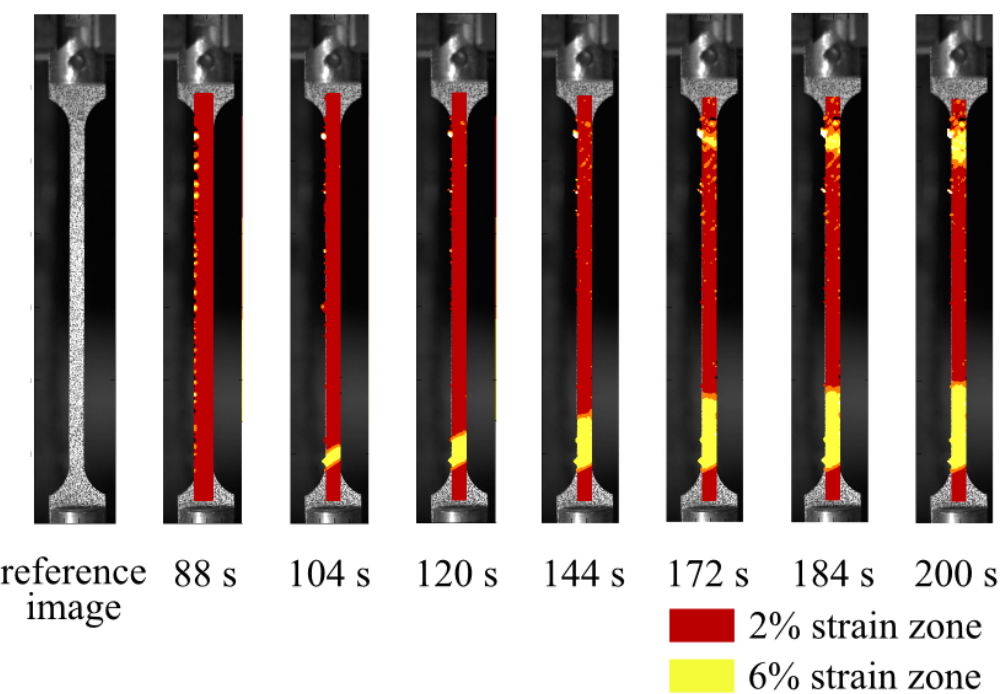

Fig. 3. Strain maps for quasi-static tensile test performed at $0.01 \mathrm{~mm} / \mathrm{s}$

In order to show more clearly the growing rate of transformed region, space-time figures are plotted. Such a space-time figure is given in figure 4(a). It is obtained as the time-evolution of the mean longitudinal strain along the deformed specimen. This mean is done over the whole width of the specimen. This was considered, contrary to the choice of a single line without meaning procedure, in order to smooth the results but it induces quantitative errors locally if the transformation front is not perpendicular to the axis of the specimen. This is supposed to be negligible and does not contradict the results on growth rate that is the point of this paper because the angle between the localised bands and the direction of the tensile loading is supposed to be constant in time ([4]).

The observation of figure 4(a) shows at a first stage a growth of a first transformed area initiated at the lower side of the specimen. Then, at a time of around $160 \mathrm{~s}$, a second transformed region appears at the upper side of the specimen. Longitudinal strain rate can also be obtained by time derivation (here a backward single time step is used). The result for the $0.01 \mathrm{~mm} / \mathrm{s}$ tensile test is given in figure $4(\mathrm{~b})$. One can observe that (i) the maximum of strain rate is obviously located at the supposed transformation 
front, (ii) that two transformations fronts are moving, in opposite directions in this case, (iii) that anywhere else the tranformation fronts, strain rate is almost zero confirming that macrosopic strain rate is due to localized phenomena only. One can noticed that the transformation region seems to have a thickness which doesn't exist in reality. The discretization of the map and the display mode used in MATLAB $^{\mathfrak{C}}$ cause this effect.

(a)

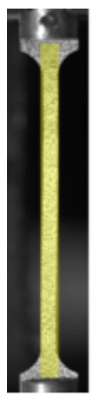

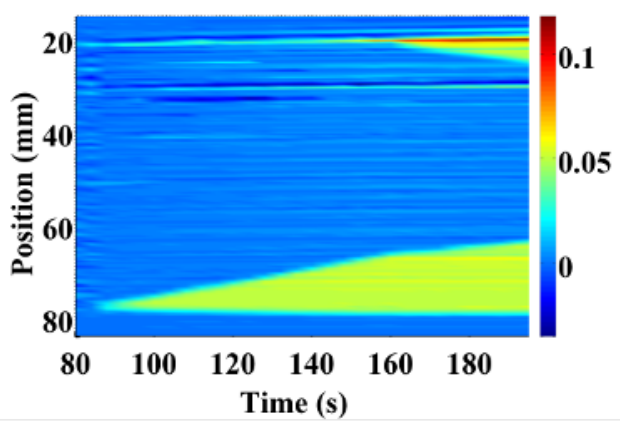

(b)

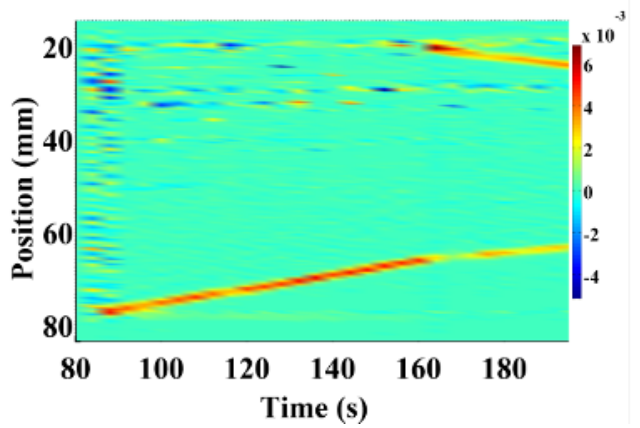

Fig. 4. Quasi-static tensile test at $0.01 \mathrm{~mm} / \mathrm{s}$. (a) Mean longitudinal strain along the specimen versus time, (b) Mean longitudinal strain rate $(/ \mathrm{s})$ along the specimen versus time.

The results of the IR measurements are not given here but the conclusions are that the maximum temperature measured in the localized exothermic domains follows exactly the same path as non zerostrain rate associated to the transformation front.

\section{Dynamic tensile experiments at $1000 \mathrm{~mm} / \mathrm{s}$}

Comparing the stress/strain response obtained in dynamic tensile test with the ones in quasi-static tensile test confirms the strain rate dependence of the macroscopic response that is observed in the litterature. This can be seen on figure 5 .

The space-time map of DIC results is given in figure 6. The spatial coordinates are given on the deformed configuration. In the same way as for static experiments and in spite of a noise level that is higher, two different levels of strain are visible at about $6 \%$ strain and about $2 \%$ strain. In the continuity of the validation procedure used in the former paragraph, it is supposed that these two levels correspond respectively to elastic deformation of austenite and full SIM. Moreover, the history of deformation occurs in four successive stages:

1. No macroscopic deformation,

2. Homogeneous elastic deformation of initial austenite phase. One can notice that homogeneity is not very good and some bands are already visible. The authors cannot state what is the origin of these measurements.

3. Appearance of first localization at the upper part of the specimen.

4. Appearance of second localization, only time-shifted of about $0.4 \mathrm{~ms}$. The two transformation fronts are then propagating, once again in opposite directions due to the geometrical constraints.

On figure 6, a dashed line has been plotted in order to separate the map in two regions. For time before $2.3 \mathrm{~ms}$, there is only tensile loading in the specimen due to the tensile wave coming from the input bar. After $2.3 \mathrm{~ms}$, reflection of the transmitted wave at the free end of the output bar changes the boundary conditions so that macroscopic strain rate in the specimen be zero.

This map constitute an original result thanks to DIC computations. Observations of martensitic transformation in dynamic tensile test has been reported in [11] but the $x-t$ diagram was extract from 
the result of several gauges positionned along the specimen. In our case, DIC allows us to evaluate the strain field on every points of the specimen, not only on localised points. Considering the fact that, as it was mentionned in [4], when the strain rate increases, the number of nucleation site can increase, DIC let us know if a local raise of the strain is due to the apparition of a new nucleation site or the propagation of existing one. This checking is crucial in evaluating the enlargement velocity.

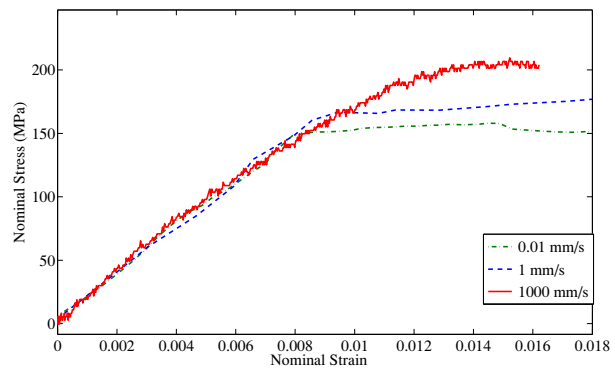

Fig. 5. Effect of prescribed velocity on the tensile curve.

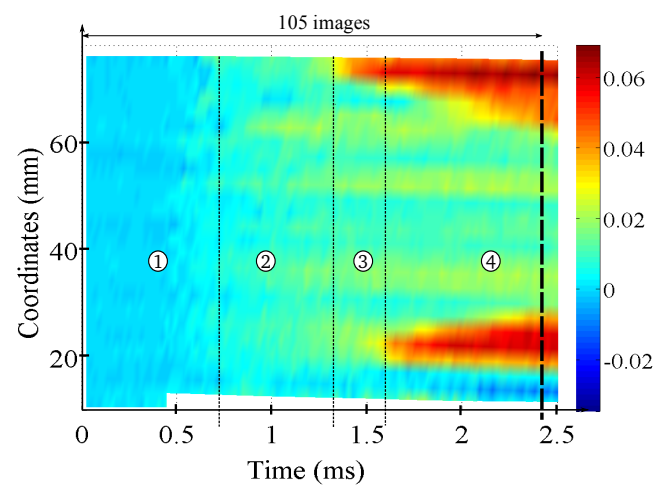

Fig. 6. Evolution of the longitudinal nominal strain - Dynamic tensile test at $1000 \mathrm{~mm} / \mathrm{s}$

\section{Measurement of the effect of prescribed velocity on the SIM growth rate}

Now that the transformed zone is clearly identified using DIC computations, it is possible to extract from the strain maps of each test the evolution of the lenght of martensite over time. This can be achieved by choosing a criterion which determines which part of the map can be considered as a transformed zone or not. In this case, a local point of the field was considered as transformed if its strain was superior to 0.04 . Using a routine, it is possible to measure, at each step of time of the test, the lenght of stress-induced martensite. By plotting this length on a curve in time, the slope appears to be the velocity of enlargement of the transformed area. The results are given in table 1 . Here, for each expected prescribed velocities, the enlargement velocity is given and the ratio between these two values is computed.

Table 1. Velocities measured using DIC techniques for a 0.04 criterion

\begin{tabular}{lllc}
\hline Expected Prescribed Velocity & Measured Prescribed Velocity & Enlargement Velocity & Ratio \\
\hline $0.01 \mathrm{~mm} / \mathrm{s}$ & $0.01 \mathrm{~mm} / \mathrm{s}$ & $0.187 \mathrm{~mm} / \mathrm{s}$ & 18.7 \\
$1 \mathrm{~mm} / \mathrm{s}$ & $0.76 \mathrm{~mm} / \mathrm{s}$ & $12.5 \mathrm{~mm} / \mathrm{s}$ & 16.5 \\
$1000 \mathrm{~mm} / \mathrm{s}$ & $1100 \mathrm{~mm} / \mathrm{s}$ & $19300 \mathrm{~mm} / \mathrm{s}$ & 17.5 \\
\hline
\end{tabular}

The ratio between measured prescribed velocity of the specimen and enlargement velocity of the transformed aera can be considered as quite constant. This proportionality between these two quantities was found in [3] for strain rate applied from $4 \times 10^{-5} s^{-1}$ to $4 \times 10^{-2} s^{-1}$. So, this study brings complementary results to these observations. 


\section{Results and conclusion}

Specific measurement techniques have been implemented in order to analyze the influence of nominal strain rate on the growth rate of the SIM region in a NiTi specimen. Hopkinson bar measurement technique, DIC applied to quasi-static and dynamic experiment, and IR thermography for static experiments give accurate and relevant results. After synchronizing DIC and IR measurements in time and space, a superimposition can be done and results show that the temperature peak, as expected, follows the transformation front. Finally, the velocity of the propagation front is measured and we show that it depends linearly on the prescribed velocity. As a conclusion, in the range of strain rates investigated in this paper, no strain rate sensitivity is observed.

\section{References}

1. E.A. Pieczyska, S.P. Gadaj, W.K. Nowacki, H. Tobushi, Exp. Mech. 46,(2006) 531-542.

2. S. Nemat-Nasser, J.Y. Choi, Acta Materialia 53,(2005) 449-454.

3. J.A. Shaw, S. Kyriakides, J. Mech. Phys. Solids, 43,(1995) No.8 1243-1281.

4. J.A. Shaw, S. Kyriakides, Acta Mater., 45,(1997) No.2 683-700.

5. Brinson, Schmidt, Lammering, J. Mech. Phys. Solids, 52, (2004) 1549-1571.

6. E.D.H. Davies, S.C. Hunter, J. Mech. Phys. Solids,11, (1963) 55-179.

7. H. Zhao, G. Gary, J. Mech. Phys. Solids,45, (1997) No.7 1185-1202.

8. G.H. Staab, A. Gilat, Exp. Mech., 31, (1991) No.3 232-235.

9. G. Besnard, F. Hild, S. Roux, Exp. Mech., 46, (2006) No.6 789-804.

10. I. Elnasri, S. Pattofatto, H. Zhao, H. Tsitsiris, F. Hild, Y. Girard, J. Mech. Phys. Solids, 55 (2007) 2652-2671.

11. J. Niemczura, K. Ravi-Chandar, J. Mech. Phys. Solids, 54 (2006) 2136-2161. 